Research article

Open Access

\title{
Construction of an MUC-1 promoter driven, conditionally replicating adenovirus that expresses the sodium iodide symporter for gene therapy of breast cancer
}

\author{
Miguel A Trujillo1, Michael J Oneal², Julia Davydova ${ }^{3}$, Elizabeth Bergert ${ }^{1}$, Masato Yamamoto ${ }^{3}$ and \\ John C Morris III1
}

\author{
1Department of Internal Medicine, Division of Endocrinology, Diabetes, Metabolism, Nutrition, Mayo Clinic Rochester, 200 First Street SW, Rochester, \\ MN 55905, USA \\ 2Department of Molecular Medicine, Mayo Clinic Rochester, 200 First Street SW, Rochester, MN 55905, USA \\ ${ }^{3}$ Department of Surgery, University of Minnesota, MMC195, 420 Delaware St., SE Minneapolis, MN 55455, USA
}

Corresponding author: John C Morris, morris.john@mayo.edu

Received: 16 Apr 2009 Revisions requested: 28 May 2009 Revisions received: 26 Jun 2009 Accepted: 27 Jul 2009 Published: 27 Jul 2009

Breast Cancer Research 2009, 11:R53 (doi:10.1186/bcr2342)

This article is online at: http://breast-cancer-research.com/content/11/4/R53

(c) 2009 Trujillo; licensee BioMed Central Ltd.

This is an open access article distributed under the terms of the Creative Commons Attribution License (http://creativecommons.org/licenses/by/2.0), which permits unrestricted use, distribution, and reproduction in any medium provided the original work is properly cited.

\begin{abstract}
Introduction The sodium iodide symporter (NIS) directs the uptake and concentration of iodide in thyroid cells. This in turn allows radioiodine imaging and therapy for thyroid cancer. To extend the use of NIS-mediated radioiodine therapy to other types of cancer, we successfully transferred and expressed the sodium-iodide symporter (NIS) gene in prostate, colon, and breast cancer cells both in vivo and in vitro by using nonreplicating adenoviral vectors.
\end{abstract}

Methods To improve virotherapy efficiency, we developed a conditionally replicating adenovirus (CRAd) in which the transcriptional cassette RSV promoter-human NIScDNA-bGH polyA was also inserted at the $\mathrm{E} 3$ region. The $E 1$ a gene is driven by the tumor-specific promoter MUC-1 in the CRAd Ad5AMUCH_RSV-NIS.
Results In vitro infection of the MUC-1-positive breast cell line T47D resulted in virus replication, cytolysis, and release of infective viral particles. Conversely, the MUC-1-negative breast cancer cell line MDA-MB-231 was refractory to the viral cytopathic effect and did not support viral replication. The data indicate that Ad5AMUCH_RSV-NIS activity is stringently restricted to MUC-1-positive cancer cells. Radioiodine uptake was readily measurable in T47 cells infected with Ad5AMUCH_RSV-NIS 24 hours after infection, thus confirming NIS expression before viral-induced cell death.

Conclusions This construct may allow multimodal therapy, combining virotherapy with radioiodine therapy to be developed as a novel treatment for breast and other MUC1-overexpressing cancers.

\section{Introduction}

The American Cancer Society's most recent estimates for breast cancer in the United States for 2009 are as follows: 192,370 new cases of invasive breast cancer and 40,170 deaths of breast cancer. Breast cancer is the most common cancer among women in the United States, other than skin cancer. It is the second leading cause of cancer death in women, after lung cancer [1]. Breast cancer is particularly dif- ficult to treat when it metastasizes and becomes resistant to antiestrogen therapies [2].

The sodium iodide symporter (NIS) is a transmembrane glycoprotein that mediates uptake of iodide into cells, especially thyroid follicular cells $[3,4]$. The presence of NIS on the basolateral membrane of thyroid cells has been exploited for many years for diagnostic imaging purposes, as well as for ablative therapy of differentiated thyroid cancer by using radi-

Ad5: adenovirus serotype 5; bGH: bovine growth hormone; BIS-TRIS: bis(2-hydroxyethyl)iminotris(hydroxymethyl)methane; CAR: coxsackievirus adenovirus receptor; CIP: calf intestine phosphatase; CPE: cytopathic effect; CRAd: conditionally replicating adenovirus; EDTA: ethylenediaminetetraacetic acid; HBSS: Hanks' buffered salt solution; LTR: long terminal repeat; MOI: multiplicity of infection; MTS: [3-(4,5-dimethylthiazol-2-yl)-5-(3carboxymethoxyphenyl)-2-(4-sulfophenyl)-2H-tetrazolium; MUC-1: mucin-1; NIS: sodium iodide symporter; PBS: phosphate-buffered saline; PCR: polymerase chain reaction; polyA: polyadenylation signal; RSV: Rous sarcoma virus; RT: room temperature; TBS-T: Tris-buffered saline Tween-20. 
oactive iodide (131I). This noninvasive therapy has proven to be a safe and effective treatment for thyroid cancer, even in advanced, metastatic disease $[5,6]$. To extend the use of NISmediated radioiodine therapy to other types of cancer, we successfully transferred and expressed the sodium-iodide symporter (NIS) gene in prostate, colon, and breast cancer cells, both in vivo and in vitro, by using adenoviral vectors. Our experience with adenovirus-mediated NIS transfer and radioiodine therapy was confirmed in a large animal model and has culminated in the opening of a phase I trial for prostate cancer that is currently in progress [7-12].

MUC-1 is a highly glycosylated transmembrane mucin. Although MUC-1 is expressed at very low levels in normal tissues; it is overexpressed by most carcinomas, including breast cancers [13]. Enhanced expression of MUC-1 is regulated mostly at the level of transcription [14]. The pronounced differential expression of MUC-1 in tumor versus normal tissues has been used in experimental developments of antitumor therapies, including MUC-1 vaccines and MUC-1 promoterrestricted antitumor-specific viruses [15-17].

All gene-therapy approaches depend on the ability to deliver therapeutic genes to target cells. However, the limited ability to transduce tumors efficiently with effective levels of therapeutic transgenes has been identified as the fundamental barrier to effective cancer gene therapy $[18,19]$. To address this issue, conditionally replicating viruses, including adenovirus, have been constructed, and their efficacy, evaluated [20-22].

Our approach to the current problems associated with virotherapy/gene therapy has been the development of tumor-specific, conditionally replicating adenoviral vectors that also harbor the NIS gene. We report here the development of a conditionally replicating adenovirus (CRAd) in which the E1a gene is driven by the tumor-specific promoter MUC-1. In addition, the transcriptional cassette RSV promoter-hNIScDNAbGH polyA was inserted at the E3 region. Our results suggest that this CRAd may represent a novel approach to breast cancer gene therapy.

\section{Materials and methods Cell culture}

The breast cancer cell lines T47D and MDA-MB-231 were used to examine CRAd Ad5AMUCH_RSV-NIS specificity. Adenovirus infection was performed for 4 hours in serum-free media. Cells were then washed once with PBS and replenished with fresh culture media. The human embryonic kidney cell line stably expressing E1A (HEK 293) was obtained from Cell Biolabs, Inc., San Diego, CA. Cells were cultured as described $[8,23]$.

\section{CRAd construct and cell lines}

The E1A gene flanked by a 5' blunt end, and an EcoRI 3' end was isolated from plasmid pCD512_F2 (a gift of Dr. Richard
Vile, Mayo Clinic) and cloned into the Promega vector pGL3Basic to yield pGL3E1. The MUC-1 promoter (a gift of Dr. Sandra Gendler, Mayo Clinic Arizona) was PCR amplified by using the forward primer AAAAAGGTACCGGACCCTAGGGTTCATCGGAG and the reverse primer AAAAAAGATCTGATTCAGGCAGGCGCTGGCTGC from the plasmid pcDNA3 [8]. The resulting PCR fragment $0.726 \mathrm{~Kb}$ spanning nt2188 to nt2914 was cloned into pGL3E1 to yield pGL3MUCH. The MUC-1 promoter-E1A-SV40 PolyA fragment was excised from pGL3MUCH by digestion with EcoRI/Kpnl and cloned into the shuttle vector pVQA-PB-NIS [23] restricted by Kpnl/ EcoRI and dephosphorylated with CIP to generated pVQA$\mathrm{MUCH}$. The human NIS cDNA under Rous Sarcoma Virus LTR promoter control was subcloned into an Ad5 genomic vector containing an E3-deleted version (ViraQuest, North Liberty, IA, USA). The pVQAMUCH shuttle vector and the NIScontaining Ad5 genomic plasmid were recombined to yield the CRAd Ad5AMUCH_RSV-NIS. Plaques were grown, purified, and characterized by ViraQuest as previously described [23]. The replication-incompetent Muc promoter-driven luciferase expression vector, AdEasyMucLuc, was constructed through homologous recombination in Escherichia coli by using the AdEasy system [24]. This vector has the transgene cassette in the $\mathrm{E} 1$ deleted region of an adenoviral vector backbone. The vector was constructed as follows: the MUC_1 promoter was derived from a plasmid pGL3MUCH and cloned into plasmid pShuttleGL3B [25] by using Kpnl and Hindlll sites. The resultant plasmid, pShuttleMuc_GL3B, was linearized with Pmel digestion and subsequently co-transformed into $E$. coli BJ5183 with the pAdEasy-1 Ad backbone plasmid. After selection of recombinants in these bacteria, the recombinant DNA was linearized with Pacl digestion and transfected into 293 cells to generate AdEasyMucLuc. The virus was propagated in 293 cells, dialyzed in phosphate-buffered saline (PBS) with $10 \%$ glycerol, and stored at $-80^{\circ} \mathrm{C}$. Titering was performed with a plaque-forming assay by using 911 cells and optical density-based measurement.

\section{MUC-1 flow cytometry}

The $10^{6}$ exponentially growing cells were washed with PBS containing $1 \mathrm{mmol} / \mathrm{L}$ EDTA and resuspended in PBS. Cells were incubated with $20 \mu \mathrm{g} / \mathrm{ml}$ mouse anti-human MUC-1 antibody (Cell Signaling Technology Inc. Beverly, MA, USA) 30 min at RT. Controls cells were processed equally, except that they were incubated with $20 \mu \mathrm{g} / \mathrm{ml}$ mouse anti-human $\left(\operatorname{lgG}_{1}\right)$ TNP anti-isotype antibody control (BD Biosciences Pharmingen, SanDiego, CA, USA). Cells were then washed in PBS and incubated with FITC-conjugated goat anti-mouse $\lg G$ secondary antibody (BD Biosciences Pharmingen) (1:100) for 30 min at RT. Cells were then washed, resuspended in PBS, and immediately analyzed with a Becton Dickinson FACScan by using CELLQuest software. 


\section{Protein isolation and Western blots}

For all Western blots, the protein concentration was measured in triplicate, and two gels were run. The first was developed with silver staining by using the PlusOne Silver Staining Kit, Protein (GE Healthcare Bio-Sciences, Uppsala, Sweden) according to the manufacturer's instruction and quantitated by using the ImageJ software [26]. This gel served as loading control (not shown). The second gel was blotted onto nitrocellulose and Western blotted. E1a and hexon extracts were prepared from $4 \times 10^{6}$ cells grown on $100-\mathrm{mm}$ tissue-culture plates and infected at MOI 20. At noted time points, cells were rinsed twice in HBSS before lysis in $1 \mathrm{ml}$ of the complete lysis$M$ reagent with supplied protease inhibitors (Roche Applied Science, Indianapolis, IN, USA) on ice with shaking for 5 minutes and then quantified by using the DC protein assay (BioRad Hercules, CA, USA). Membrane proteins extracts were prepared for NIS and CAR Western blotting as described [27]. Lysate aliquots, $1 \mu \mathrm{g}$ of protein, were electrophoresed by using I a X cell II Blot Module (Invitrogen, Carlsbad, CA, USA) on $10 \%$ BIS-TRIS polyacrylamide gels under reducing conditions at $200 \mathrm{~V}$ for 1 hour. Proteins were then blotted to nitrocellulose membranes at $25 \mathrm{~V}$ for 1 hour and processed with the ECL advanced Western blot detection kit (GE Healthcare, Uppsala, Sweden) according to the manufacturer's instructions. E1A primary antibody (Santa Cruz Biotechnology, Santa Cruz, CA, USA) was diluted 1:20,000, and the anti-Ad5 hexon lgG (Santa Cruz Biotechnology), 1:100,000. Anti-CAR analyses were performed by running $5 \mu \mathrm{g}$ of membrane protein or $1 \mu \mathrm{g}$ of cell extract on a $10 \%$ Bis-Tris polyacrylamide gels under nonreducing conditions. Blocking steps were performed as described earlier. Blots were incubated with primary mouse Anti-CAR, clone RmcB (Upstate Cell Signaling Solutions Inc., Danvers, MA, USA). The antibody was diluted $1: 10,000$ in TBS-T with $0.1 \%$ blocking reagent for 90 minutes. NIS Western blots were performed as previously described [27].

\section{Cytopathic effect assays}

The $1 \times 10^{6}$ cells were infected at MOI 1 . At noted time points, the cells were photographed under a light microscope at $100 \times$ magnification.

\section{MTS assay}

The $5 \times 10^{4}$ cells were seeded in 96-well tissue-culture plates and incubated at $37^{\circ} \mathrm{C}, 5 \% \mathrm{CO}_{2}$ in triplicate. Twenty-four hours after plating, the cells were infected at the indicated $\mathrm{MOI}$. The virus-containing medium was exchanged with fresh medium, and at each time point, $50 \mu$ of CellTiter 96 Aqueous One Solution Cell Proliferation Assay (Promega Corporation, Madison, WI, USA) was applied and then incubated at $37^{\circ} \mathrm{C}$, $5 \% \mathrm{CO}_{2}$ for 2 hours. Absorbance was then read at $490 \mathrm{~nm}$ by using a plate spectrophotometer.

\section{Replication assay of the CRAd vector}

The $4 \times 10^{6}$ cells were plated in $150-\mathrm{cm}^{2}$ culture flasks and infected at MOI 1 and MOI 0.1. Seventy-two hours after infection, virus was harvested from media and cells separately and purified by using the ViraBind Adenovirus Purification Kit (Cell Biolabs, San Diego, CA, USA). Virus titers were quantified by plaque assay on HEK 293 cells.

\section{I uptake}

Uptake of $125 \mathrm{l}$ by virus-infected cells was measured as described $[10,28]$. In brief, $1 \times 10^{6}$ cells/well were plated on 36-well tissue-culture plates and infected at $\mathrm{MOI} 0,0.5,5,10$, 20 , and 50. At indicated time points, iodide-uptake measurement was performed in triplicate in HBSS supplemented with

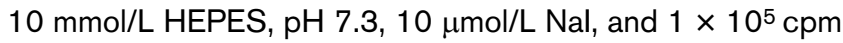
of ${ }^{125} \mathrm{l}$. Control wells were incubated in the same buffer plus $10 \mu \mathrm{mol} / \mathrm{L} \mathrm{KClO}_{4}$. Plates were gently mixed and incubated at $37^{\circ} \mathrm{C}, 5 \% \mathrm{CO}_{2}$, for 45 minutes. After incubation, buffers were aspirated and the cells gently washed once with $4^{\circ} \mathrm{C} 10$ $\mathrm{mmol} / \mathrm{L}$ HEPES, pH 7.3 buffer. Cells were lysed in $1 \mathrm{ml} 1 \mathrm{~N}$ $\mathrm{NaOH}$ with shaking for 20 minutes at RT, and lysates were assayed for ${ }^{125}$ I on a gamma counter.

\section{Noninvasive imaging of xenografted tumors}

All experimental protocols were reviewed and approved by the Mayo Clinic Institutional Animal Care and Use Committee (IACUC). Xenografts derived from the T47D cell line were established on each flank of 6-week-old athymic nude Foxn1 nu mice (Harlan, Madison, WI, USA) by subcutaneous injection of $4 \times 10^{6}$ cells resuspended in $0.125 \mu \mathrm{l}$ media and $0.125 \mu$ l of BD Matrigel basement membrane matrix (BD Biosciences, Bedford, MA). Mice were maintained on a low-iodine diet and $\mathrm{T}_{4}$ supplementation ( $5 \mathrm{mg} / \mathrm{L}$ ) in their drinking water throughout the duration of the experimentation to maximize radioisotope uptake in the tumor and minimize uptake by the thyroid [8-10]. The mice were examined daily for tumor growth. Tumor volume was measured with calipers twice weekly and calculated by the formula:

$$
\text { Volume }=\mathrm{L} \times \mathrm{H} \times \mathrm{W} \times 0.51
$$

When tumors reached $100 \mathrm{~mm}^{3}$, mice received one intratumoral (IT) injection of $10^{11} \mathrm{vp}$ of the AdAMUCH_RSV-NIS CRAd on the left flank; the right flank served as control. Two days later, an intraperitoneal dose of $0.5 \mathrm{mCi}$ of ${ }^{123}$ sodium iodide was given, and 1 hour later, radioiodine imaging was performed by using a $\gamma$-camera equipped with a low-energy high-resolution collimator (VG System; GE Healthcare, Milwaukee, WI). Regions of uptake were quantified and expressed as a fraction of the total amount of the applied radioiodine. 


\section{Results Virus construction}

The conditionally replicating adenoviruses (CRAd) Ad5AMUCH_RSV-NIS harbors the transcriptional cassette RSV promoter-human NIScDNA-bGH polyA inserted at the E3 region. In this CRAd, the E1a gene is driven by a $0.726-\mathrm{Kb}$ version of the tumor-specific MUC-1 promoter spanning nt2188 to nt2914 [17] (Figure 1).

\section{MUC-1 and CAR expression}

Two breast cancer cell lines, T47D and MDA-MB-231, were characterized for their expression of MUC-1. No difference in the flow-cytometry histogram was found between the isotype control and the MUC-1 antibody in MDA-MB-231, confirming that this cell line is MUC-1- (data not shown). We then zeroed the FACScan by using this cell line and analyzed the T47D under this new setting. This analysis revealed that T47D is MUC-1 positive, whereas MDA-MB-231 is MUC-1 negative (Figure 2a). Moreover, when these two cell lines were infected with the nonreplicating virus AdEasyGL3B-Muc, in which the Luc gene is driven by the MUC-1 promoter, only the T47D cell line supported MUC-1-driven Luc activity, whereas MDA-MB231 was completely negative (Figure $2 b$ ). To exclude the possibility that inability to support viral replication was due to lack of CAR-receptor expression, CAR-receptor expression was assayed with Western blot of membrane extracts prepared from the two cell lines. To control for protein loading, identical amounts of protein were loaded on a second gel that was silver stained for protein assessment (not shown). The insert in Figure $2 \mathrm{a}$ supports the notion that both cell lines readily express the CAR receptor at the cell plasma membrane. We conclude that T47D is MUC-I positive, whereas MDA-MB-231 is MUC-1 negative, and that both cell lines express CAR and thus are capable of being infected by wild-type capsid Ad5.

\section{Conditional replication, oncolysis, and spread of Ad5AMUCH_RSV-NIS}

We examined the specificity of Ad5AMUCH_RSV-NIS replication by using several methods. First, we examined the specificity of viral proteins expression. MUC-1-driven E1A and Hexon expression were examined with Western blotting.
T47D and MDA-MB-2321 cells were infected with Ad5AMUCH_RSV-NIS at MOI 20. Figure 3 shows two major differences between the permissive T47D cell line and the nonpermissive MDA-MB-231. Only cells infected with Ad5AMUCH_RSV-NIS expressed viral proteins. Most important, a marked difference was noted in the quality and quantities of viral protein expressed in the permissive cell compared with the nonpermissive cell line (Figure 3a). We also examined hexon expression, a product of late protein synthesis and a very good indicator of viral assembly [29]. Here again, the amount of hexon protein produced 48 hours after infection in the permissive cell line T47D outperformed that produced in nonpermissive cell lines MDA-MB-231 (Figure 3b).

Cells infected with Ad5PB_RSV-NIS at MOI 1 were monitored for viral-induced cytopathic effect (CPE) by light microscopy. Four days after infection, the morphology of the MDA-MB-231 cells was unchanged; however, clear signs of CPE in T47D are detected under the same conditions of infection (Figure 4).

To address the issue of virus specific cell killing, the permissive cell line T47D was infected with the Ad5AMUCH_RSVNIS CRAd at increasing MOls and then assayed daily by MTS. The results were compared with those obtained with the nonpermissive cell line MDA-MB-231. The T47D cells showed a reduced viability that was both time and dose dependent (Figure $5 \mathrm{a}$ ). Conversely, MDA-MB-231 viability was completely refractory to virus-mediated cell killing (Figure $5 \mathrm{~b}$ ).

Virus-progeny production after infection with the Ad5AMUCH_RSV-NIS CRAd was measured in T47D, and MDA-MB-231 cells 3 days after infection at $\mathrm{MOI} 1$ or 0.1 by burst assay (Figure 6). To distinguish between released and intracellular virus, media and cell were separated, and virus was purified separately from each. The amount of virus produced by the T47D cells was 6 orders of magnitude greater than the amount produced by the MDA-MB-231 cells.

Taken together, these data demonstrate that replication of the Ad5AMUCH_RSV-NIS virus is stringently restricted to MUC-

Figure 1

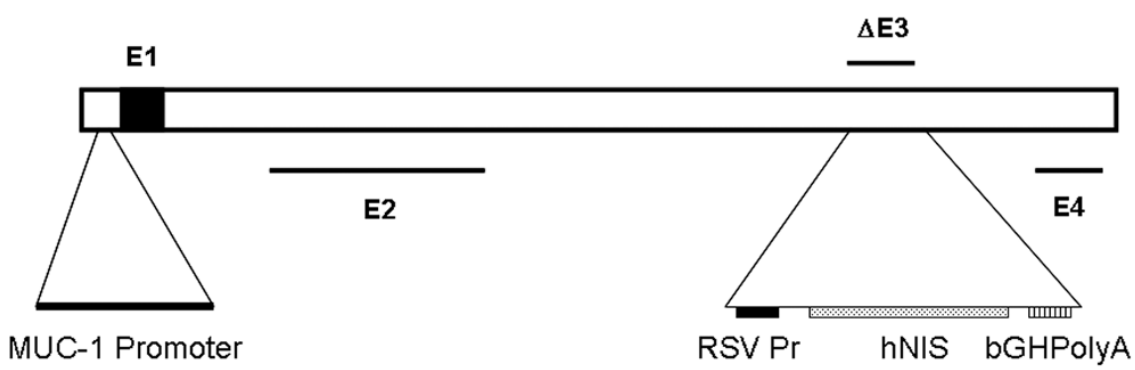

Structure of the Ad5AMUCH_RSV-NIS CRAd. The E1A gene is driven by the tumor-specific mucin-1 (MUC-1) promoter. The Rous sarcoma virus (RSV) promoter drives expression of the sodium iodide symporter (NIS) gene inserted at the E3 region. 
Figure 2
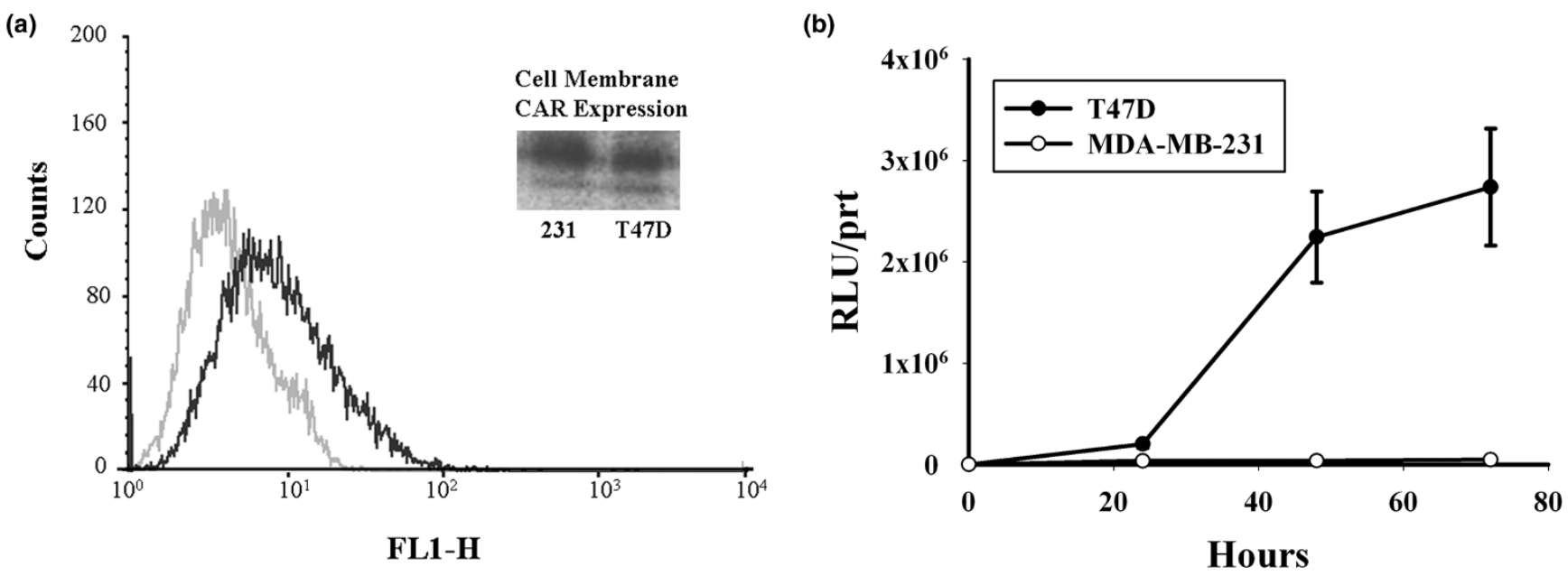

Mucin-1 (MUC-1) and coxsackievirus adenovirus receptor (CAR) expression. (a) Relative membrane MUC-1 expression in T47D (black line) and MDA-MB-231 cells (gray line) was measured with flow cytometry. Insert: Western blot with an anti-CAR receptor antibody of membrane proteins extracted from T47D and MDA-MB-231 cells. (b) MUC-1 promoter expression was monitored in T47D and MDA-MB-231 by measuring MUC-1driven expression of luciferase activity after transduction with the nonreplicating virus AdEasyGL3B-Muc.

1-positive breast cancer cells, defining this virus as a conditionally-replicating adenovirus or CRAd.

\section{NIS expression and radioiodine uptake}

Expression of the NIS protein was examined with Western blotting by using a mouse monoclonal anti-human NIS antibody [30]. Figure 7a shows that T47D and MDA-MB-231 cells transfected with the Ad5PB_RSV-NIS CRAd expressed NIS, which was detected as a broad band spanning from $130 \mathrm{Kd}$ to $110 \mathrm{kDa}$. Visualization of the NIS band as a smear is expected; it reflects differences in NIS glycosylation [30,31]. Expression of the NIS gene in the nonpermissive cell line
MDA-MB-231 is supported by the RSV ubiquitous promoter and shows that this cell line is susceptible to Ad5 infection.

Having shown that infected cells are capable of expressing membrane-bound NIS, we next investigated whether the protein was functional. Dose-response and kinetic curves of iodine uptake were constructed. Infection of T47D cells with Ad5AMUCH_RSV-NIS at MOI 20 showed maximal uptake at 24 hours after infection, followed by a twofold decrease in 125 uptake at later time points (Figure 7b). Radioiodine uptake was inhibited by $\mathrm{KClO}_{4}$, a well-known inhibitor of NIS activity [3]. We infer from this result that an optimal time window exists for NIS-mediated radioiodine uptake in the permissive cells, after

Figure 3
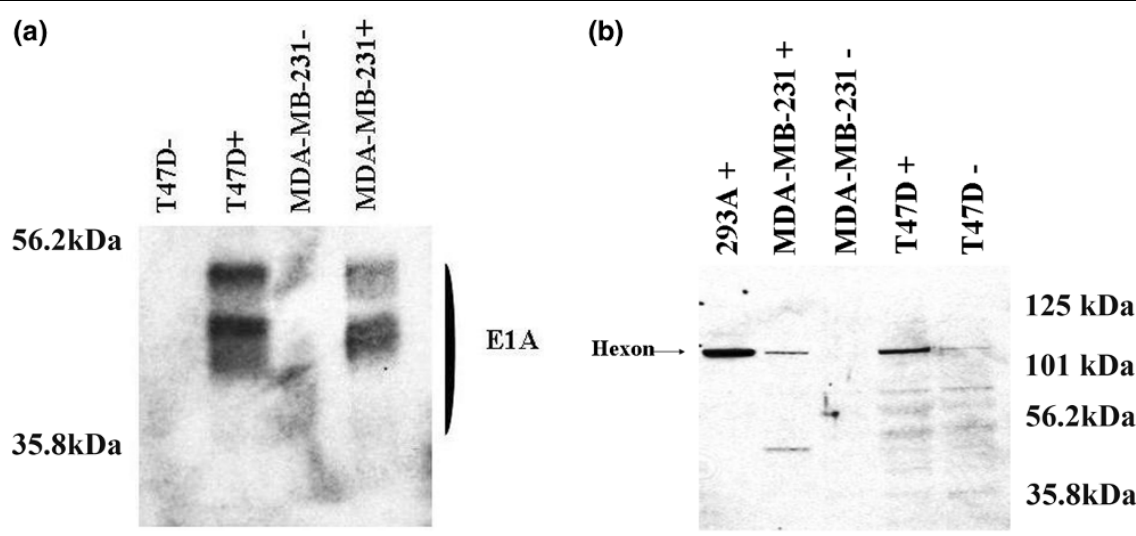

Viral proteins expression. Expression of E1A (a) was assayed in T47D and MDA-MB-23) cells. Cells were infected with Ad5AMUCH_RSV-NIS at multiplicity of infection (MOI) 20 , and extracts were prepared at 24 hours after infection and compared with extracts from noninfected cells. (b) Hexon expression was assayed in MDA-MB-231 and T47D 48 hours after infection and compared with uninfected cells. HEK 293 cells infected with Ad5AMUCH_RSV-NIS were used as control. 
Figure 4

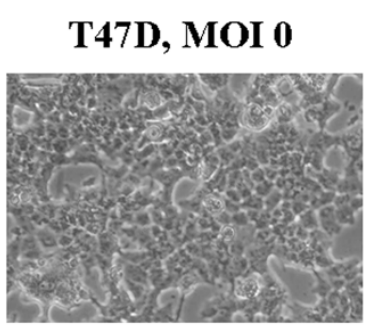

MDA-MB-231, MOI 0
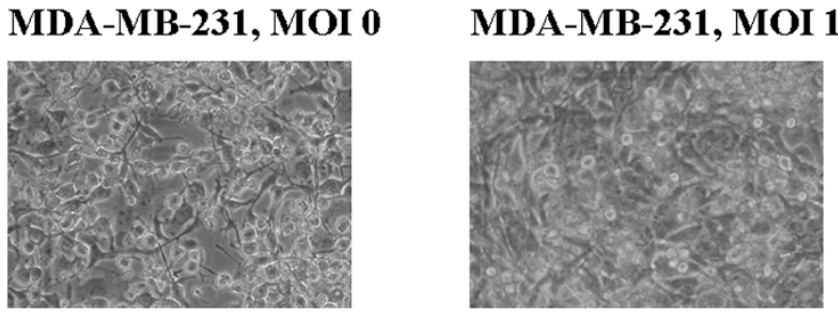

Cytopathic effect. Ad5PB_RSV-NIS-induced cytopathic effect was assessed with light microscopy 4 days after infection in T47D and MDA-MB-231 cells.

which, uptake, and as a consequence, NIS expression declines. This might be due to virus replication and induction of CPE. Twenty-four hours after infection, a radioiodine uptake dose-response to increasing $\mathrm{MOI}$ concentrations was built. Figure 7c shows that radioiodine uptake was linear over the range of $\mathrm{MOI}$ used in the experiment.

Having shown in cell culture that Ad5AMUCH_RSV-NIS can infect, multiply, and direct NIS expression in permissive cells, we wished to confirm that viral infection and NIS expression also take place in vivo. Figure 8 reveals strong ${ }^{123}$ uptake by the T47D tumor that has been injected with the Ad5PB_RSVNIS CRAd 48 hours after infection. Quantitation of the image yielded an uptake in infected tumors of more than $30 \%$ of the injected dose at 1 hour. The stomach and thyroid also were imaged because of native NIS expression, and the bladder, because of tracer excretion [9].

Taken together our results demonstrate that Ad5AMUCH_RSV-NIS directs NIS expression in permissive cells before the onset of cell lysis.

\section{Discussion}

Although a number of gene-therapy approaches have been developed for many cancers, clinical trials completed to date have fallen short of expectations [32-36], largely due to limited tumor transduction, and this limitation has been defined as the most important fundamental barrier to effective cancer gene therapy [37]. Our approach to addressing the problem of low tumor transduction is to allow vector replication by using conditionally replicating adenovirus.

We used a transcriptional-regulation method placing the $E 1 A$ gene under control of the tumor-specific promoter MUC-1. The rationale for this choice is based on the observation that transcriptional-dependent regulation seems to be the most efficient strategy for CRAd development [38]. In addition, we inserted the NIS gene under control of the powerful but nonspecific RSV promoter. We used a truncated version of the MUC-1 promoter that was previously shown to be specifically active in MUC-1-positive breast cancer cell lines [17]. The rationale and utility for use of this promoter is that more than $90 \%$ of breast tumors overexpress the MUC-1 gene [13]. By this method, viral replication, and consequently extended NIS

\section{Figure 5}
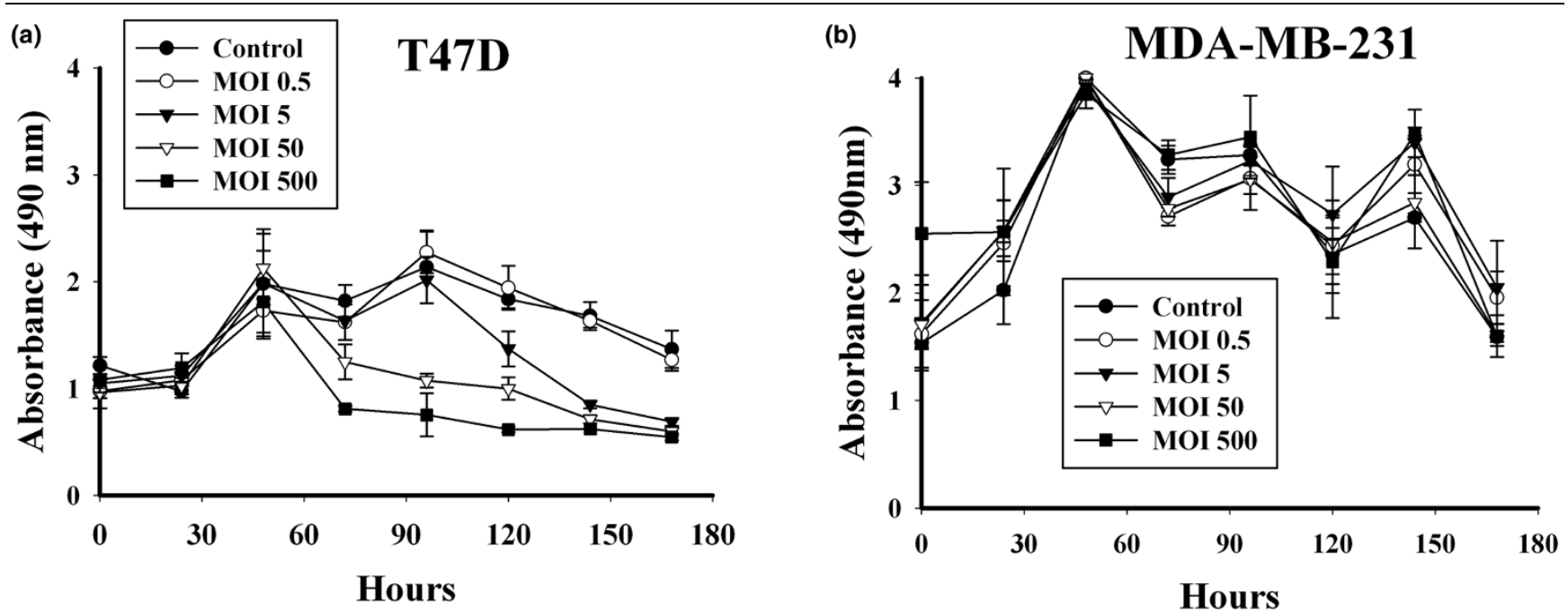

Cell-proliferation assay. (a) T47D and (b) MDA-MB-231. Cells were infected with Ad5PB_RSV-NIS, and cell viability was monitored daily by using MTS assay. 
Figure 6

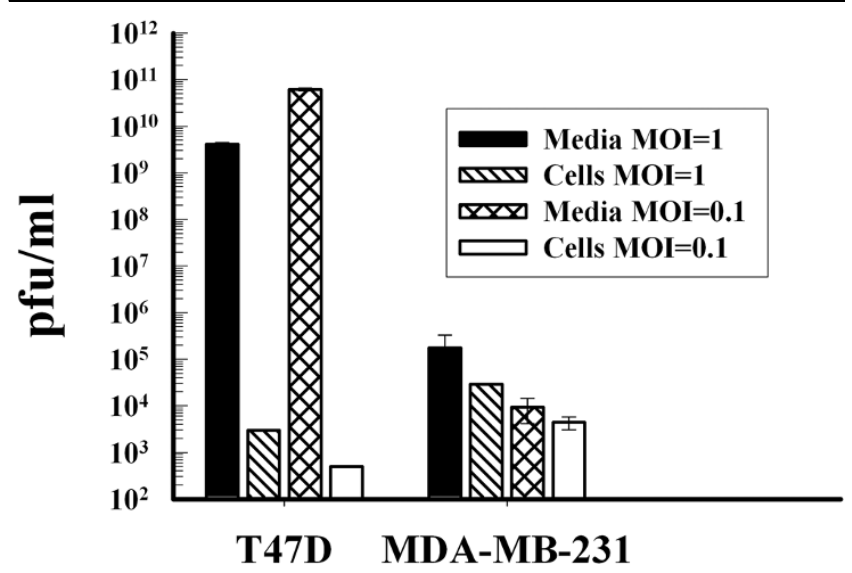

Cell-specific replication of Ad5AMUCH_RSV-NIS. T47D and MDAMB-231 cells were infected with Ad5AMUCH_RSV-NIS at multiplicity of infection (MOI) 1 or $\mathrm{MOI} 0.1$. Viral progeny were isolated from media and cells and quantitated with plaque assay on 293 cells. expression, will target a wide spectrum of MUC-1-positive breast tumors.

In the present study, we confirmed that this MUC-1 promoter version restricts viral protein synthesis, cytopathic effect, cell lysis, and infectious viral progeny production to MUC-1-positive breast cancer cells. Moreover, the bulk of viral progeny produced by the MUC-1-positive breast cancer cell line T47D was found in the media, indicating that viral infection of T47D cells with the Ad5AMUCH_RSV-NIS CRAd resulted in cell lysis and subsequent release of infectious viral particles. We hypothesize that the release of infectious viral particles should allow wide viral spread within tumors. Virus specificity was demonstrated by using the MUC-1-negative breast cancer cell line MDA-MB-231. The MDA-MB-231 cell line expresses cellsurface CAR receptor because we visualized CAR expression in membrane-bound electrophoresed proteins. It supports limited $\mathrm{E} 1 \mathrm{~A}$ and hexon expression and supports NIS expression. All these data confirm that MDA-MB-231 is infected by the Ad5AMUCH_RSV-NIS, yet it is resistant to CRAd-induced

Figure 7

(a)

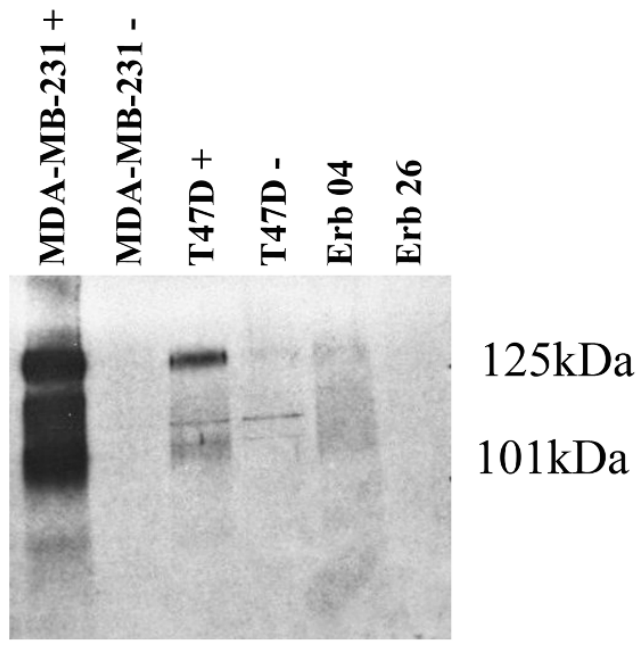

(b)

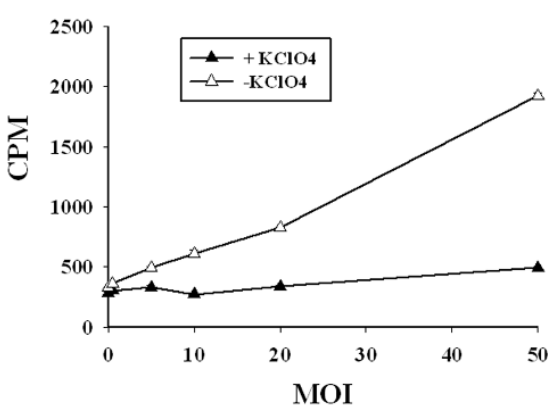

(c)

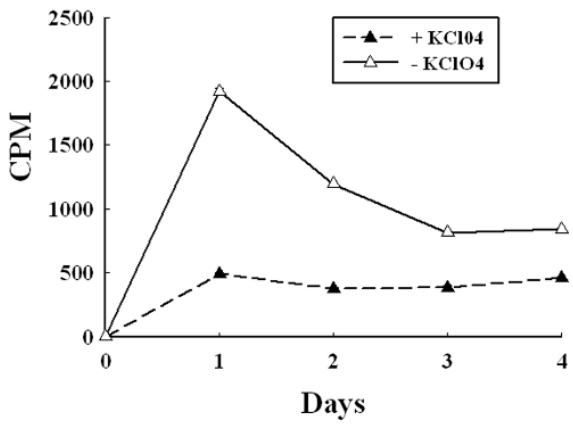

Analysis of sodium iodide symporter (NIS) expression. (a) NIS expression assayed with Western blotting in Ad5AMUCH_RSV-NIS-infected cells. Membrane proteins were prepared 24 hours after infection. (b) Radioiodine uptake dose-response. T47D cells were infected at the indicated multiplicity of infection (MOI). Radioiodine uptake was measured 24 hours after infection. (c) Kinetics of NIS-mediated radioiodine uptake. T47D cells were infected at MOI 20 with the Ad5AMUCH_RSV-NIS conditionally replicating adenovirus (CRAd), and ${ }^{125}$ uptake was measured daily. 
Figure 8

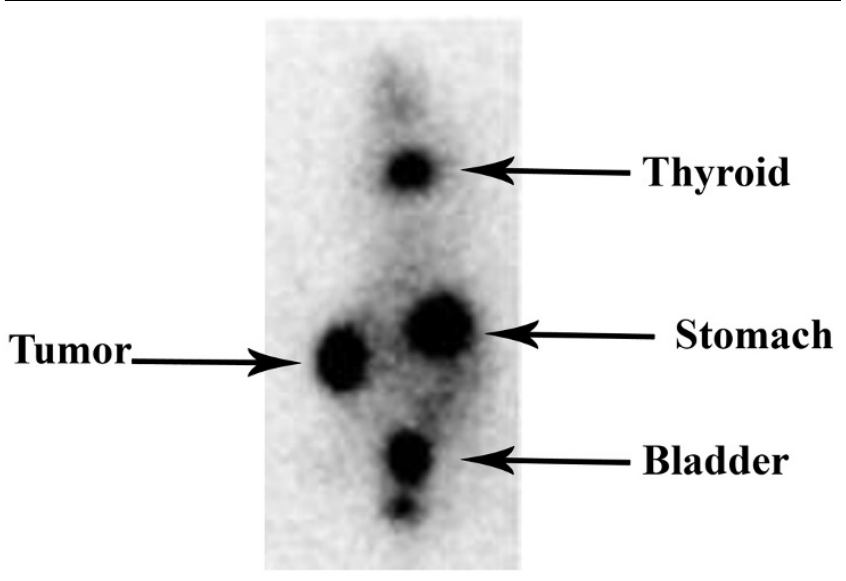

Radioiodine imaging of T47D xenografted tumors. T47D xenografted tumors were established in athymic nude mice. The left-flank tumor was injected with $10^{11} \mathrm{vp}$ of the Ad5AMUCH_RSV-NIS CRAd, and 48 hours later, $0.5 \mathrm{mCi}$ of ${ }^{123}$ I was administered intraperitonially. Images were captured using a $\gamma$ camera 1 hour after radioisotope administration.

oncolysis or viral replication or both. These data demonstrate that the Ad5AMUCH_RSV-NIS CRAd is stringently dependent on MUC-1 overexpression and should not replicate within normal tissue, where MUC1 expression is minimal.

The NIS protein must be properly targeted to the cell plasma membrane to be functional [39]. Our results also showed that the Ad5AMUC_RSV-NIS CRAd directs correct NIS expression and thus radioiodine uptake in vitro and in vivo. Under the control of the RSV promoter, the NIS protein was correctly targeted to the cell plasmamembrane, thereby promoting high levels of iodine uptake in T47D cells. Our results also show that in MUC-1-negative CAR-positive breast cancer tumors, the NIS gene also can be expressed, because it is under the control of the ubiquitous RSV promoter. Here, the cell line MDA-MB-231 showed very strong NIS protein expression by Western blot, which translated to robust radioiodine uptake (data not shown). Hence the Ad5AMUCH_RSV-NIS CRAd could be used as a non-replicating NIS-expressing vector.

However, in this case, NIS gene expression was transient, thus narrowing the window for radioiodine therapy and loss of the combined radiovirotherapy.

A discrepancy is apparent in the kinetics of NIS expression and luciferase expression that can be explained by several influencing factors; First, the /uciferase gene was cloned at the E1 region of a nonreplicating adenovirus, under the control of the MUC-1 promoter, whereas the NIS gene was cloned at the E3 region of a replicating adenovirus under the RSV promoter. Second, the stability of two very different mRNAs and proteins cannot be assumed to be equal.
Finally, NIS is an integral membrane protein, whereas luciferase remains cytoplasmic. Therefore, the pattern of expression of luciferase and NIS under these conditions is not expected to overlap precisely.

NIS is already a therapeutic gene, in that its native expression in thyroid cells is used for therapy for thyroid cancer and hyperthyroidism, where its efficacy is very high [6]. NIS expression could represent an ideal therapeutic gene for breast cancer therapy, because it allows a large bystander effect. Because of the physics of ${ }^{131}$ I decay, which occurs primarily through emission of a $\beta$ particle that traverses up to $2 \mathrm{~mm}$ within the tissue. Thus, not every cell must be transfected and express NIS to be affected by radioiodine therapy. This is critical to the effectiveness of any cancer gene-therapy strategy because of the difficulty in achieving $100 \%$ transfection with the therapeutic gene, even in the case of a replicating virus. Moreover, NIS expression can be directly monitored and quantitated noninvasively. The uptake of radioiodine in tissues can be quantitated, and both the distribution and the quantity of NIS expression can be easily and safely monitored noninvasively.

The limited efficacy observed in clinical trials of cancer gene therapies to date has suggested that combinatorial therapies to treat cancer will likely be more effective and possibly required for complete tumor eradication. The hypothesis developed from this observation is that attacking tumor cells through different mechanisms of action may prevent tumor cells from developing resistance to the treatment combination and may more effectively induce immunity against the tumor [40]. We hypothesize that, because Ad5AMUC_RSV-NIS CRAd harbors the NIS gene, it should improve the therapeutic value of the CRAd by allowing multimodal therapy in a single agent combining viral-mediated tumor lysis as well as radioiodine-mediated therapy.

\section{Conclusions}

In conclusion, the features of Ad5PB_RSV-NIS CRAd address favorably the major hurdles identified in gene-therapy clinical trials for anticancer treatments. Further evaluation, including efficacy studies in animal models, is needed to determine the potential of this radio/virotherapy approach to affect breast cancer treatment.

\section{Competing interests}

The authors declare that they have no competing interests.

\section{Authors' contributions}

MAT constructed the CRAd, participated in the design of the study, performed FACS analysis, luc assay, and cytopathic effect, supervised the work of MJO and ER, and drafted the manuscript. MJO performed MTS and cell-specific replication. JD constructed, amplified, and purified AdEasyGL3B-Muc with MY. EB performed Western blots and radioiodine uptake. MY constructed, amplified, and purified AdEasyGL3B-Muc 
AdEasyGL3B-Muc with JD. JCM conceived of the study, participated in its design and coordination, and helped to draft the manuscript.

\section{Acknowledgements}

The authors thank Tracy Decklever, Azhal Ahmadi, Robert Parrish, and Dr. Jian Qiao for technical help. This work was supported by Mayo Clinic Breast Cancer Specialized Program of Research Excellence grant P50CA116201, James N. Ingle Project Director.

\section{References}

1. American Cancer Society: 2009 [http://www.cancer.org/docroot/ $\mathrm{CRI} /$ content/

CRI 22 1X How many people get breast cancer 5.asp?site area $=]$.

2. Bernard-Marty C, Cardoso F, Piccart MJ: Facts and controversies in systemic treatment of metastatic breast cancer. Onco/ogist 2004, 9:617-632.

3. Carrasco N: lodide transport in the thyroid gland. Biochim Biophys Acta 1993, 1154:65-82.

4. Jhiang SM, Cho JY, Ryu KY, DeYoung BR, Smanik PA, McGaughy VR, Fischer AH, Mazzaferri EL: An immunohistochemical study of $\mathrm{Na}+/ \mathrm{l}-$ symporter in human thyroid tissues and salivary gland tissues. Endocrinology 1998, 139:4416-4419.

5. Mazzaferri EL, Kloos RT: Carcinoma of follicular epithelium: radioiodine and other treatments and outcomes. In The Thyroid: A Fundamental and Clinical Text 7th edition. Edited by: Braverman LE, Utiger RD. Philadelphia: Lippincott Raven; 1996:922-945

6. Van Nostrand D, Wartofsky L: Radioiodine in the treatment of thyroid cancer. Endocrinol Metab Clin North Am 2007, 36:807-822.

7. Scholz IV, Cengic N, Baker $\mathrm{CH}$, Harrington $\mathrm{KJ}$, Maletz $\mathrm{K}$, Bergert ER, Vile R, Goke B, Morris JC, Spitzweg C: Radioiodine therapy of colon cancer following tissue-specific sodium iodide symporter gene transfer. Gene Ther 2005, 12:272-280.

8. Dwyer RM, Bergert ER, O'Connor MK, Gendler SJ, Morris JC: In vivo radioiodide imaging and treatment of breast cancer xenografts after MUC1-driven expression of the sodium iodide symporter. Clin Cancer Res 2005, 11:1483-1489.

9. Dwyer RM, Bergert ER, O'Connor MK, Gendler SJ, Morrris JC: Sodium lodide symporter-mediated radioiodide imaging and therapy of ovarian tumor xenografts in mice. Gene Ther 2006, 13:60-66.

10. Spitzweg C, Dietz AB, O'Connor MK, Bergert ER, Tindall DJ, Young CY, Morris JC: In vivo sodium iodide symporter gene therapy of prostate cancer. Gene Ther 2001, 8:1524-1531.

11. Dwyer RM, Schatz SM, Bergert ER, Myers RM, Harvey ME, Classic KL, Blanco MC, Frisk CS, Marler RJ, Davis BJ, O'Connor MK, Russell SJ, Morris JC: A preclinical large animal model of adenovirus-mediated expression of the sodium-iodide symporter for radioiodide imaging and therapy of locally recurrent prostate cancer. Mol Ther 2005, 12:835-841.

12. Gene therapy and radioactive iodine in treating patients with locally recurrent prostate cancer that did not respond to external-beam radiation therapy [http://clinicaltrials.gov/ct/show/ NCT00788307]

13. Gendler SJ: MUC1, the renaissance molecule. J Mammary Gland Biol Neoplasia 2001, 6:339-353.

14. Kovarik A, Peat N, Wilson D, Gendler SJ, Taylor-Papadimitriou J: Analysis of the tissue-specific promoter of the MUC1 gene. J Biol Chem 1993, 268:9917-9926.

15. Chen L, Chen D, Manome Y, Dong Y, Fine HA, Kufe DW: Breast cancer selective gene expression and therapy mediated by recombinant adenoviruses containing the DF3/MUC1 promoter. J Clin Invest 1995, 96:2775-2782.

16. Mukherjee P, Pathangey LB, Bradley JB, Tinder TL, Basu GD, Akporiaye ET, Gendler SJ: MUC1-specific immune therapy generates a strong anti-tumor response in a MUC1-tolerant colon cancer model. Vaccine 2007, 25:1607-1618.

17. Kurihara T, Brough DE, Kovesdi I, Kufe DW: Selectivity of a replication-competent adenovirus for human breast carcinoma cells expressing the MUC1 antigen. J Clin Invest 2000, 106:763-771.

18. Herrmann F: Cancer gene therapy: principles, problems, and perspectives. J Mol Med 1995, 73:157-163.

19. Waehler R, Russell SJ, Curiel DT: Engineering targeted viral vectors for gene therapy. Nat Rev Genet 2007, 8:573-587.

20. Russell SJ: RNA viruses as virotherapy agents. Cancer Gene Ther 2002, 9:961-966.

21. Bischoff JR, Kirn DH, Williams A, Heise C, Horn S, Muna M, Ng L, Nye JA, Sampson-Johannes A, Fattaey A, McCormick F: An adenovirus mutant that replicates selectively in p53-deficient human tumor cells. Science 1996, 274:373-376.

22. Markert JM, Malick A, Coen DM, Martuza RL: Reduction and elimination of encephalitis in an experimental glioma therapy model with attenuated herpes simplex mutants that retain susceptibility to acyclovir. Neurosurgery 1993, 32:597-603.

23. Anderson RD, Haskell RE, Xia H, Roessler BJ, Davidson BL: A simple method for the rapid generation of recombinant adenovirus vectors. Gene Ther 2000, 7:1034-1038.

24. He TC, Zhou S, da Costa LT, Yu J, Kinzler KW, Vogelstein B: A simplified system for generating recombinant adenoviruses. Proc Natl Acad Sci USA 1998, 95:2509-2514.

25. Yamamoto M, Alemany R, Adachi Y, Grizzle WE, Curiel DT: Characterization of the cyclooxygenase-2 promoter in an adenoviral vector and its application for the mitigation of toxicity in suicide gene therapy of gastrointestinal cancers. Mol Ther 2001, 3:385-394.

26. Rasband WS: ImageJ. 1997 [http://rsbweb.nih.gov/ij/]. United States National Institutes of Health, Bethesda, Maryland, USA

27. Kakinuma H, Bergert ER, Spitzweg C, Cheville JC, Lieber MM, Morris JC: Probasin promoter (ARR(2)PB)-driven, prostatespecific expression of the human sodium iodide symporter $(\mathrm{h}-$ NIS) for targeted radioiodine therapy of prostate cancer. Cancer Res 2003, 63:7840-7844.

28. Weiss SJ, Philp NJ, Grollman EF: lodide transport in a continuous line of cultured cells from rat thyroid. Endocrinology 1984 , 114:1090-1098.

29. Boulanger PA: Adenovirus assembly: self-assembly of partially digested hexons. J Virol 1975, 16:1678-1682.

30. Castro MR, Bergert ER, Beito TG, Mclver B, Goellner JR, Morris JC: Development of monoclonal antibodies against the human sodium iodide symporter: immunohistochemical characterization of this protein in thyroid cells. J Clin Endocrinol Metab 1999, 84:2957-2962

31. Spitzweg C, Dutton CM, Castro MR, Bergert ER, Goellner JR, Heufelder AE, Morris JC: Expression of the sodium iodide symporter in human kidney. Kidney Int 2001, 59:1013-1023.

32. Cascallo M, Capella G, Mazo A, Alemany R: Ras-dependent oncolysis with an adenovirus VAl mutant. Cancer Res 2003, 63:5544-5550.

33. Humphreys MJ, Greenhalf W, Neoptolemos JP, Ghaneh P: The potential for gene therapy in pancreatic cancer. Int $\mathrm{J}$ Pancreatol 1999, 26:5-21.

34. Hung MC, Hortobagyi GN, Ueno NT: Development of clinical trial of E1A gene therapy targeting HER-2/neu-overexpressing breast and ovarian cancer. Adv Exp Med Biol 2000, 465:171-180.

35. Miura Y, Ohnami S, Yoshida K, Ohashi M, Nakano M, Fukuhara M, Yanagi K, Matsushita A, Uchida E, Asaka M, Yoshida T, Aoki K Intraperitoneal injection of adenovirus expressing antisense K-ras RNA suppresses peritoneal dissemination of hamster syngeneic pancreatic cancer without systemic toxicity. Cancer Lett 2005, 218:53-62.

36. Young LS, Searle PF, Onion D, Mautner V: Viral gene therapy strategies: from basic science to clinical application. J Pathol 2006, 208:299-318.

37. Schmidt-Wolf G, Schmidt-Wolf IG: Human cancer and gene therapy. Ann Hematol 1994, 69:273-279.

38. Bazan-Peregrino M, Carlisle RC, Hernandez-Alcoceba R, Iggo R, Homicsko K, Fisher KD, Hallden G, Mautner V, Shen Y, Seymour LW: Comparison of molecular strategies for breast cancer virotherapy using oncolytic adenovirus. Hum Gene Ther 2008, 19:873-886.

39. Kaminsky SM, Levy O, Salvador C, Dai G, Carrasco N: The Na+/ I- symporter of the thyroid gland. Soc Gen Physiol Ser 1993, 48:251-262. 
Breast Cancer Research Vol 11 No 4 Trujillo et al.

40. Chu RL, Post DE, Khuri FR, Van Meir EG: Use of replicating oncolytic adenoviruses in combination therapy for cancer. Clin Cancer Res 2004, 10:5299-5312. 